\title{
PADRÕES ESPACIAIS DA OCORRÊNCIA DE LEISHMANIOSE VISCERAL HUMANA NA CIDADE DE NATAL-RN: A INFLUÊNCIA DAS ÁREAS DE RISCO SOCIAL
}

\section{SPATIAL PATTERNS OF THE OCCURRENCE OF HUMAN VISCERAL LEISHMANIASIS IN THE CITY OF NATAL-RN: THE INFLUENCE OF THE AREAS OF SOCIAL RISK}

\author{
Karen Kaline Teixeira \\ Núcleo de Análise de Situação de Risco em Saúde \\ Centro de Controle de Zoonoses de Natal-RN \\ karenkaka@outlook.com.br \\ Kennedy Gomes do Nascimento \\ Núcleo de Análise de Situação de Risco em Saúde \\ Centro de Controle de Zoonoses de Natal-RN \\ kennedynascimento1988@gmail.com \\ Reginaldo Lopes Santana \\ Núcleo de Análise de Situação de Risco em Saúde \\ Centro de Controle de Zoonoses de Natal-RN \\ reginaldo.lopes.santana@gmail.com \\ Ana Mayara Gomes de Souza \\ Mestre em Saúde Coletiva \\ Universidade Federal do Rio Grande do Norte - UFRN \\ ana.mayara@yahoo.com.br \\ Talita Araujo de Souza \\ Doutoranda em Ciências da Saúde pela Universidade Federal do Rio Grande do Norte - UFRN \\ Professora Substituta da Faculdade de Ciências da Saúde do Trairi - FACISA/UFRN \\ talitaaraujo23@hotmail.com \\ Isabelle Ribeiro Barbosa \\ Doutora em Saúde Coletiva, Professora da Faculdade de Ciências da Saúde do Trairi - FACISA \\ Universidade Federal do Rio Grande do Norte - UFRN \\ isabelleribeiro@oi.com.br
}

\begin{abstract}
RESUMO
Objetivou-se nesse estudo analisar a ocorrência de casos de Leishmaniose visceral humana (LVH) e sua proximidade com as áreas de risco social no município de Natal-RN. Foram analisados 285 casos confirmados de LVH registrados no SINAN no período de 2008 a 2018. Para a sobreposição dos casos e das áreas de Risco social utilizou-se a análise de Kernel. Foi calculada a distância média anual de cada ponto à área de risco mais próxima. A densidade de Kernel revelou que a ocorrência dos casos é mais predominante nas áreas periféricas, sobretudo em áreas localizadas na região Norte e Oeste e sobrepostas às áreas de risco social. A distância média entre os casos e a área de risco mais próxima foi de 445 metros. Observou-se uma redução da distância média entre os casos e as áreas de risco social a cada ano, no período de 2008 e 2013, e um aumento no período de 2014 a 2018. A menor distância média foi registrada em 2012 (227 metros) e a maior foi em 2017 (677 metros). A modelagem espacial apontou que a dinâmica da ocorrência da leishmaniose visceral no município de Natal-RN está relacionada à proximidade das áreas de risco social.
\end{abstract}

Palavras chave: Leishmaniose Visceral Humana. Análise de Kernel. Risco social. Espacialização.

Recebido em: 07/08/2019

Aceito para publicação em: 09/09/2019 


\begin{abstract}
The objective of this study was to analyze the occurrence of cases of human visceral leishmaniasis (LVH) and its proximity to the areas of social risk in the municipality of NatalRN. We analyzed 285 confirmed cases of LVH recorded in SINAN in the period from 2008 to 2018. Kernel analysis was used to overlap the cases and the areas of social risk. The average annual distance from each point to the nearest risk area was calculated. Kernel density revealed that the occurrence of cases is more prevalent in peripheral areas, mainly in areas located in the north and west, and overlapping the areas of social risk. The average distance between the cases and the closest risk area was 445 meters. There was a reduction in the mean distance between the cases and the areas of social risk each year, in the period of 2008 and 2013, and an increase in the period from 2014 to 2018 . The shortest average distance was recorded in 2012 (227 meters) and the highest was in 2017 (677 meters). Spatial modeling showed that the dynamics of the occurrence of visceral leishmaniasis in the municipality of Natal-RN is related to the proximity of the areas of social risk.
\end{abstract}

Keywords: Human Visceral leishmaniasis. Kernel analysis. Social risk. Geospatialization.

\title{
INTRODUÇÃO
}

A leishmaniose visceral (LV) é causada pelos parasitas protozoários Leishmania donovani e Leishmania infantum (= Leishmania chagasii) e é uma doença potencialmente fatal com distribuição mundial, na Ásia, África Oriental, América do Sul e região do Mediterrâneo. Clinicamente, é uma doença sistêmica crônica, caracterizada pela febre de longa duração, com perda de peso, astenia, anemia, dentre outros sintomas, e que apresenta alta taxa de letalidade em doentes não tratados, crianças desnutridas e indivíduos infectados pelo Vírus da Imunodeficiência Humana (HIV) (CAVALCANTI; VALE, 2014).

Estima-se que cerca de $90 \%$ dos 50.000 a 90.000 novos casos que surgem a cada ano ocorram nas áreas rurais da Índia, Sudão, Sudão do Sul, Quênia, Somália, Etiópia e Brasil, e que apenas $30 \%$ dos casos são relatados. Epidemias com alta mortalidade em adultos e crianças ocorrem e, em algumas áreas, foram associadas a movimentos em massa de populações não imunes de ou para áreas endêmicas, como ocorreu no Sudão nos anos 90, com centenas de milhares de vidas perdidas (DNDi, 2019). Considerada uma doença negligenciada, sua importância no contexto da saúde pública no Brasil aumentou significativamente nos últimos anos (CARDIM et al., 2016; TOLEDO et al., 2017).

No Brasil, a LV foi descrita inicialmente na década de 1930, na Região Nordeste, que concentrava 90\% dos casos registrados no país até a década de 1990. Antes caracterizada como uma doença rural típica das regiões semiáridas do nordeste brasileiro, apresentou-se em franca expansão acometendo áreas urbanas de pequeno e médio porte e, atualmente, já é registrada em grandes centros urbanos, revelando o processo de periurbanização e urbanização da doença. Entre os anos de 2006 e 2008, a transmissão autóctone da LV já estava sendo relatada em mais de 1.200 municípios em 21 Unidades Federadas (BARBOSA, 2016).

O ciclo da transmissão da LV é zoonótico, sendo o cão doméstico considerado o principal reservatório, e o flebotomíneo Lutzomyia longipalpis o vetor de maior importância epidemiológica (CARDIM et al., 2013). A expansão da LV no país está diretamente relacionada com a urbanização dos casos da doença e do vetor, demonstrando a sua plasticidade de adaptar-se a ambientes antropicamente modificados, o que sugere a ação humana como um fator significativo na expansão deste agravo em saúde. Outros fatores que contribuem para a expansão da doença é a infraestrutura sanitária precária, a criação de animais que atuam como reservatórios do parasita, condições climáticas favoráveis à proliferação do vetor, cobertura vegetal com predomínio de árvores frutíferas, crescimento desordenado em áreas urbanas com acúmulo da matéria orgânica, a migração de populações humanas e caninas de áreas endêmicas e a negligência dos primeiros sintomas da doença (D'ANDREA et al., 2017; SILVA et al., 2017).

Diante desse cenário, para conter esse avanço territorial e diminuir a morbidade e letalidade desse agravo, o Ministério da Saúde brasileiro publicou no ano de 2006 o Programa de Controle da Leishmaniose DOI: http://dx.doi.org/10.14393/Hygeia153249966 $\quad$ Hygeia 15 (31): 121 - 133, Junho/2019 página 122 
Visceral (PVCLC), com medidas baseadas no diagnóstico e tratamento precoce dos casos humanos, redução da população de flebotomíneos, eliminação de reservatórios e atividades de educação em saúde. Ressalta-se que, conforme o PVCLC, as ações de prevenção e controle adotadas pelos municípios devem estar baseadas em análises epidemiológicas fundamentadas na estratificação por risco (ZUBEN; DONALISIO, 2016).

A avaliação de doenças endêmicas na perspectiva dos determinantes ambientais e sociais da doença tem sido o enfoque de alguns estudos empregando técnicas de geoprocessamento. Analisar a vulnerabilidade de zonas específicas para a transmissão da LV humana por meio da distribuição espacial da LV urbana pode contribuir para o melhor entendimento do processo de transmissão heterogêneo, resultante da organização socioterritorial de um município. A importância da aplicação destas técnicas é a identificação de aglomerados espaciais e espaço-temporais para a identificação de áreas com maior risco de ocorrência do agravo. Estes, uma vez identificados, podem ajudar os serviços de saúde a priorizá-los, tanto em termos de vigilância como de controle, no sentido de evitar sua ocorrência futura ou de minimizála (CHIARAVALOTTI-NETO, 2017).

O estado do RN possui ambiente urbano propício à ocorrência de LV, com um clima e topografia que favorecem para a proliferação do vetor e habitações em precárias condições sócio-sanitárias. As taxas de letalidade por LV registradas no Rio Grande do Norte têm estado acima da média nacional nos últimos anos (BARBOSA, 2013), passando de 3,4\%, em 1994, para 5,7\%, em 2009, o que representou incremento de $67,6 \%$. Um dos principais fatores que contribuem para o aumento dessa letalidade é o diagnóstico tardio (BRASIL, 2011). O município de Natal, capital do estado, é considerado uma área endêmica para LV e classificada pelo Ministério da Saúde como uma região de transmissão intensa da doença, onde se observa a persistência de velhas áreas de ocorrência e surgimento de novos focos, o que demonstra dificuldades no seu controle e aponta a necessidade de estudos sobre sua distribuição e a relação com áreas e populações sob-risco.

Assim, diante da magnitude desse agravo e considerando que os números dos casos da LV ainda se apresentam em grandes proporções, considerando que estudos de distribuição e análise espacial têm sido ferramentas úteis o para o fortalecimento e redirecionamento das políticas públicas existentes e, mais diretamente, à vigilância em saúde, o objetivo deste trabalho foi analisar por meio da análise espacial, a ocorrência de casos de Leishmaniose visceral humana $(\mathrm{LVH})$ e sua proximidade com as áreas de risco social no município de Natal-RN.

\section{MÉTODOS}

Trata-se de um estudo ecológico para identificação de áreas de risco para a Leishmaniose visceral humana na cidade de Natal, capital do estado do Rio Grande do Norte, região Nordeste do Brasil. O município de Natal possui uma área de $167,2 \mathrm{~km}^{2}$, com densidade demográfica de $4.808,20 \mathrm{hab} / \mathrm{km}^{2} \mathrm{e}$ estimativa populacional de 877.662 habitantes. A cidade registra temperatura média de $28^{\circ} \mathrm{C}$, clima úmido com chuvas regulares no período de março a julho. O município é dividido em 36 bairros, distribuídos em quatro regiões administrativas (Norte, Sul, Leste e Oeste), com diferentes características territoriais, físicas, demográficas e de infraestrutura. Todo o território da cidade está inserido em área urbanizada, não possuindo área rural (SEMURB, 2017).

Os casos de LV utilizados foram obtidos a partir do trabalho de rotina de vigilância epidemiológica dos casos humanos de LV realizado pelo Centro de Controle de Zoonoses da cidade de Natal. A pesquisa foi produzida utilizando dados de 285 casos confirmados de Leishmaniose visceral humana ocorridos em Natal no período de janeiro de 2008 a dezembro de 2018, a partir de dados secundários registrados no Sistema de Informação de Agravos de Notificação (SINAN).

Os casos de LVH estiveram distribuídos no Distrito Sul (29 casos), Distrito Norte I ( 81 casos), Distrito Norte II (82), no Distrito Oeste (54 casos) e no Distrito Leste (39 casos), sendo todos registrados na área urbanizada. Os campos 'logradouro' e 'número' foram utilizados para realização do georreferenciamento. Os casos foram georreferenciados manualmente no Google Earth e Maps para identificação das coordenadas $(\mathrm{x}, \mathrm{y})$ em graus decimais e posteriormente adicionados ao programa QGIS 2.12 Lyon (ORACLE AMERICA, INC. CALIFORNIA, 2008). 
Figura 01: Localização do município de Natal-RN

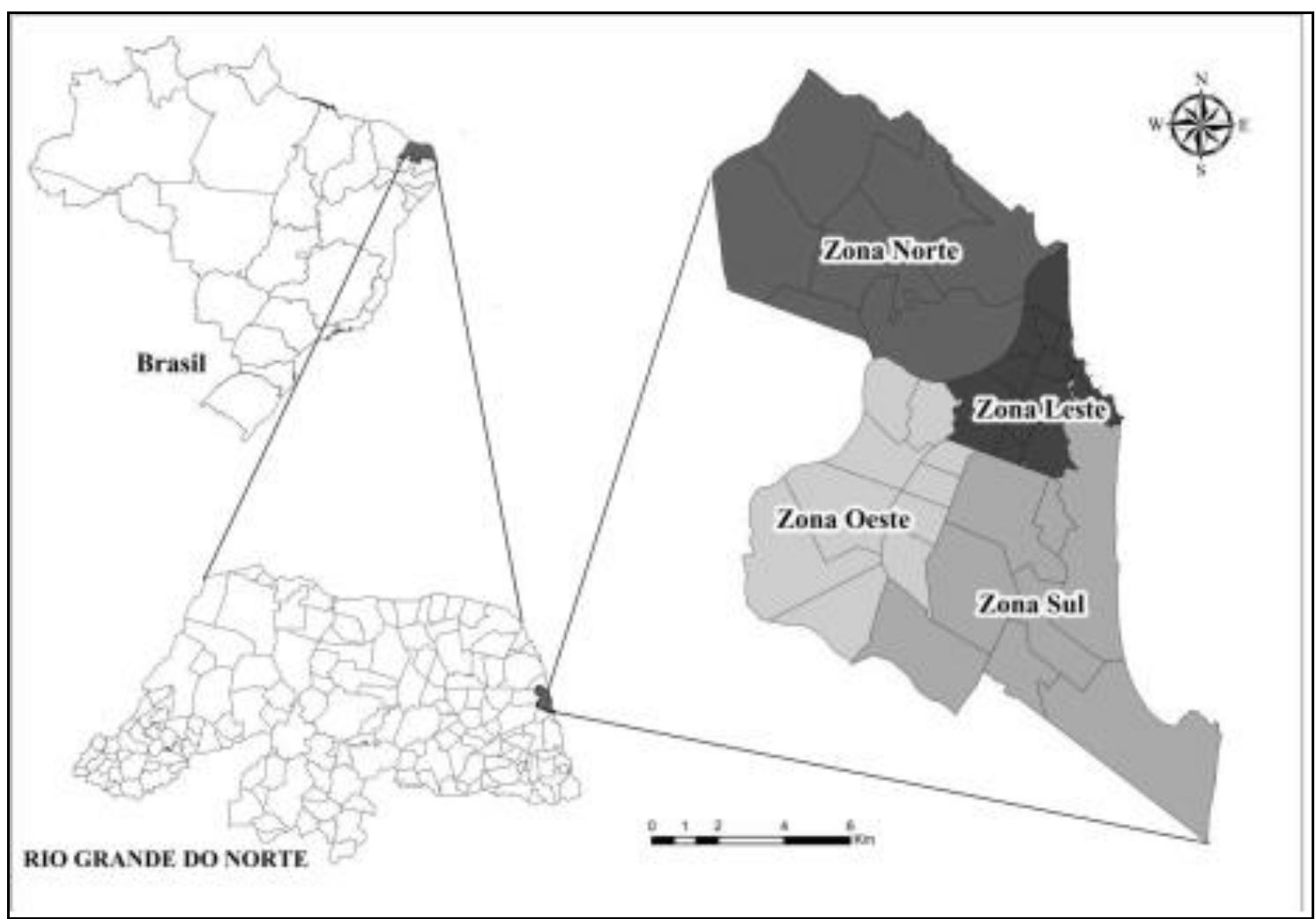

A variável socioambiental utilizada neste estudo, denominada como 'Áreas de Risco Social', foi desenvolvida pela Secretaria de Meio Ambiente e Urbanismo de Natal-RN, que em sua composição considerou aspectos geológicos, hidrológicos e antrópicos. Na metodologia para identificação de áreas de risco, foram considerados a existência dos seguintes processos geradores de risco, indicativos de ocupações irregulares ou invasões: (a) Deslizamento de solo; (b) Queda de Barreira; (c) Carreamento de Lixo; (d) Erosão "Voçoroca / Assoreamento" (encostas ou falésias); (e) Inundação (áreas naturalmente favoráveis ao acúmulo d'água ou com drenagem insuficiente); (f) Alagamento pluvial; (g) Ocupação irregular de Faixa de Domínio (áreas de domínio de estradas, ferrovias, linhas de transmissão ou gasodutos); (h) Área de Preservação Permanente (irregularmente ocupadas). A partir da presença ou ausência dessas características, o Índice de Risco foi calculado e as áreas de risco identificadas, como podem ser visualizadas na Figura 2. O mapa produzido a partir dessa classificação foi denominado de Mapa Social, entendido como um instrumento focalizador da pobreza na cidade (SEMURB, 2008).

Para a identificação das áreas de risco para ocorrência da LVH e sua sobreposição com a variável socioambiental, foram realizadas análises de estatística espacial, que permitiram estimar as densidades desses eventos dentro da área de estudo, utilizando o estimador de densidade Kernel. Foi aplicado o estimador de densidade de Kernel, sendo utilizada neste estudo a função de alisamento Quártica (biponderada) com 100, 200 e 300 metros de largura de banda e grade regular composta por $500 \times 322$ células. A escolha dos raios foi determinada quanto à preconização do Ministério da Saúde para as ações de controle da doença (BRASIL, 2006). O Kernel de intensidade permite estimar a quantidade de eventos por unidade de área, em cada célula de uma grade regular que recobre a região estudada. Essa técnica não paramétrica, além de estimar a intensidade da ocorrência de casos em toda a superfície analisada, permite filtrar a variabilidade de um conjunto de dados, ao mesmo tempo em que retém suas principais características locais (SILVERMAN, 1988). Os resultados foram apresentados em mapas, cujas áreas foram classificadas com níveis de densidade que variaram de acordo com a cor e tonalidade. 
Karen Kaline Teixeira Kennedy Gomes do Nascimento Reginaldo Lopes Santana

Padrões espaciais da ocorrência de leishmaniose visceral humana na cidade de Natal-RN: a influência das áreas de risco social
Ana Mayara Gomes de Souza

Talita Araujo de Souza

Isabelle Ribeiro Barbosa

Para a verificação da proximidade entre os casos e as áreas de risco social, foi utilizado os recursos target_layer no QGIS para cada um deles, obtendo vizinho mais próximo entre cada ponto e a área de risco mais próxima ao caso. Realizou-se uma vetorização de pontos. O cálculo da distância de cada ponto foi realizado no software QGIS 2.12. A partir da medida de distância de cada ponto, foi calculada a distância média anual (DMA), sendo calculado pelo somatório das distâncias de cada ponto à área de risco social mais próxima, dividido pelo número de pontos, em cada ano.

Figura 02: Delimitação das áreas de Risco social no município de Natal-RN

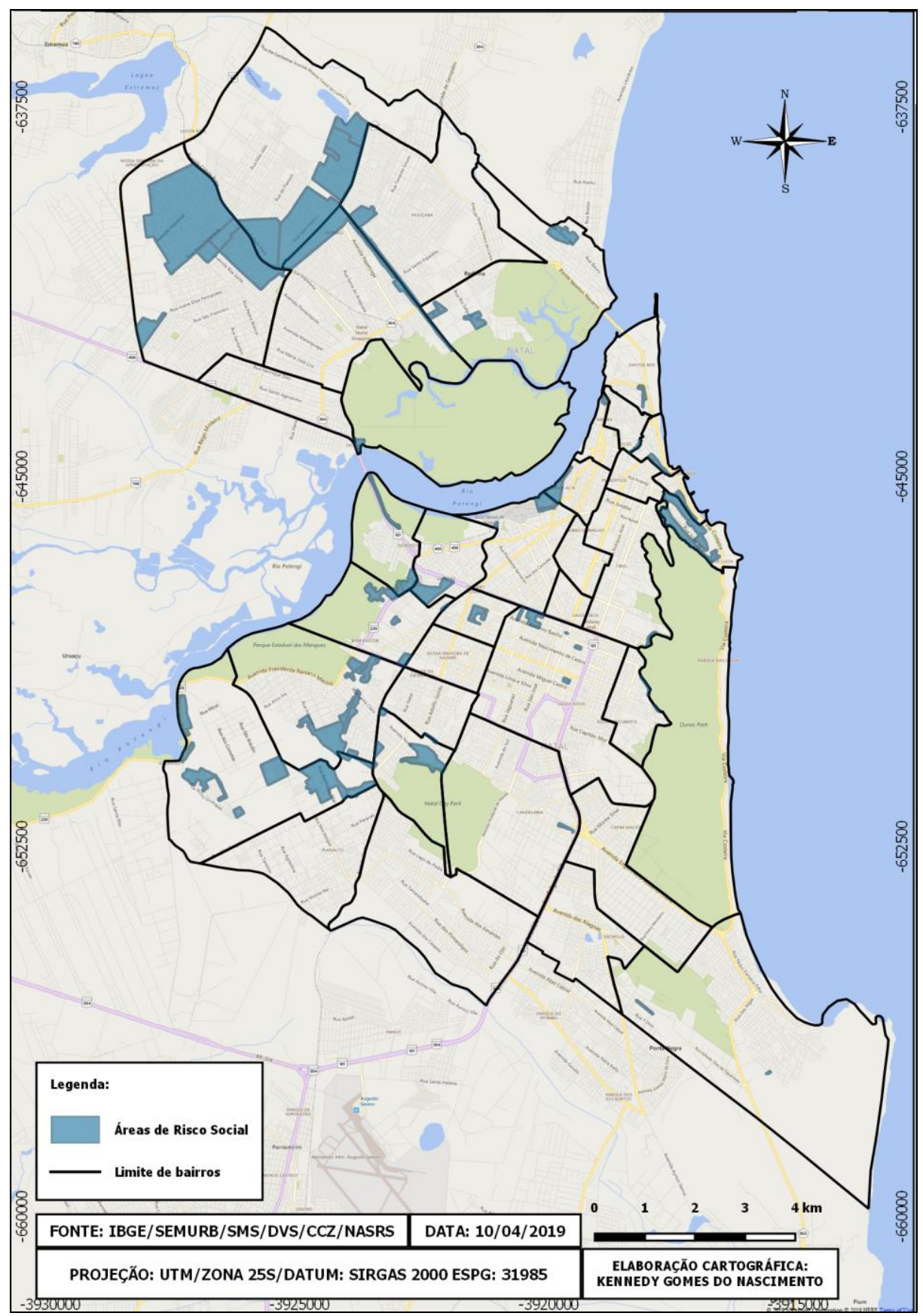


Karen Kaline Teixeira

Kennedy Gomes do Nascimento Reginaldo Lopes Santana

Padrões espaciais da ocorrência de leishmaniose Ana Mayara Gomes de Souza

Talita Araujo de Souza visceral humana na cidade de Natal-RN: a influência das áreas de risco social

Isabelle Ribeiro Barbosa

\section{RESULTADOS}

Os resultados do presente estudo demonstram que as maiores densidades de Kernel para a ocorrência dos casos é mais predominante nas áreas periféricas no período de 2008 a 2011, sobretudo em áreas localizadas nas regiões Norte e Oeste da cidade. A partir de 2012, observa-se a expansão da doença para outros focos na cidade, nos distritos Lestes e Sul. Para todos os anos estudados, fica evidente a sobreposição entre a ocorrência dos casos e as áreas de risco social (Figura 3).

Figura 03: Mapa de densidade Kernel dos casos de Leishmaniose visceral humana sobrepostos às áreas de Risco social no município de Natal-RN dos anos de 2008 a 2018
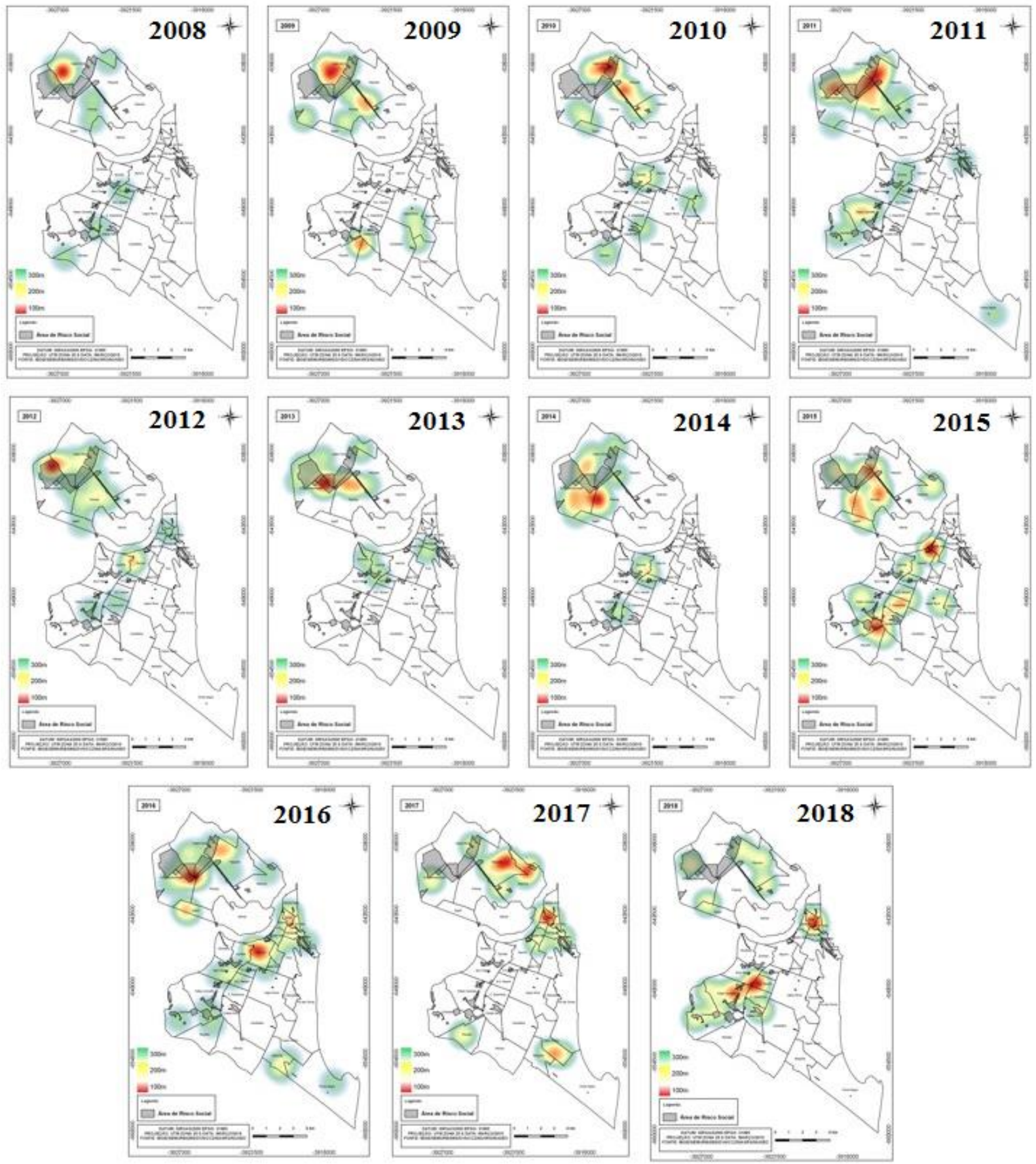
Karen Kaline Teixeira Kennedy Gomes do Nascimento Reginaldo Lopes Santana

Padrões espaciais da ocorrência de leishmaniose visceral humana na cidade de Natal-RN: a influência das áreas de risco social

Ana Mayara Gomes de Souza

Talita Araujo de Souza

Isabelle Ribeiro Barbosa

$\mathrm{Na}$ figura 4, que traz a localização de todos os casos de LVH do período de 2008 a 2018, observamse duas grandes manchas de sobreposição: no distrito Norte, uma mancha de maior intensidade, sobreposta à grande área de risco social presente nesse distrito; a segunda área caracteriza-se como um corredor que se estende do distrito Leste ao distrito Oeste.

Figura 04: Análise das áreas de risco para a ocorrência da Leishmaniose Visceral Humana.

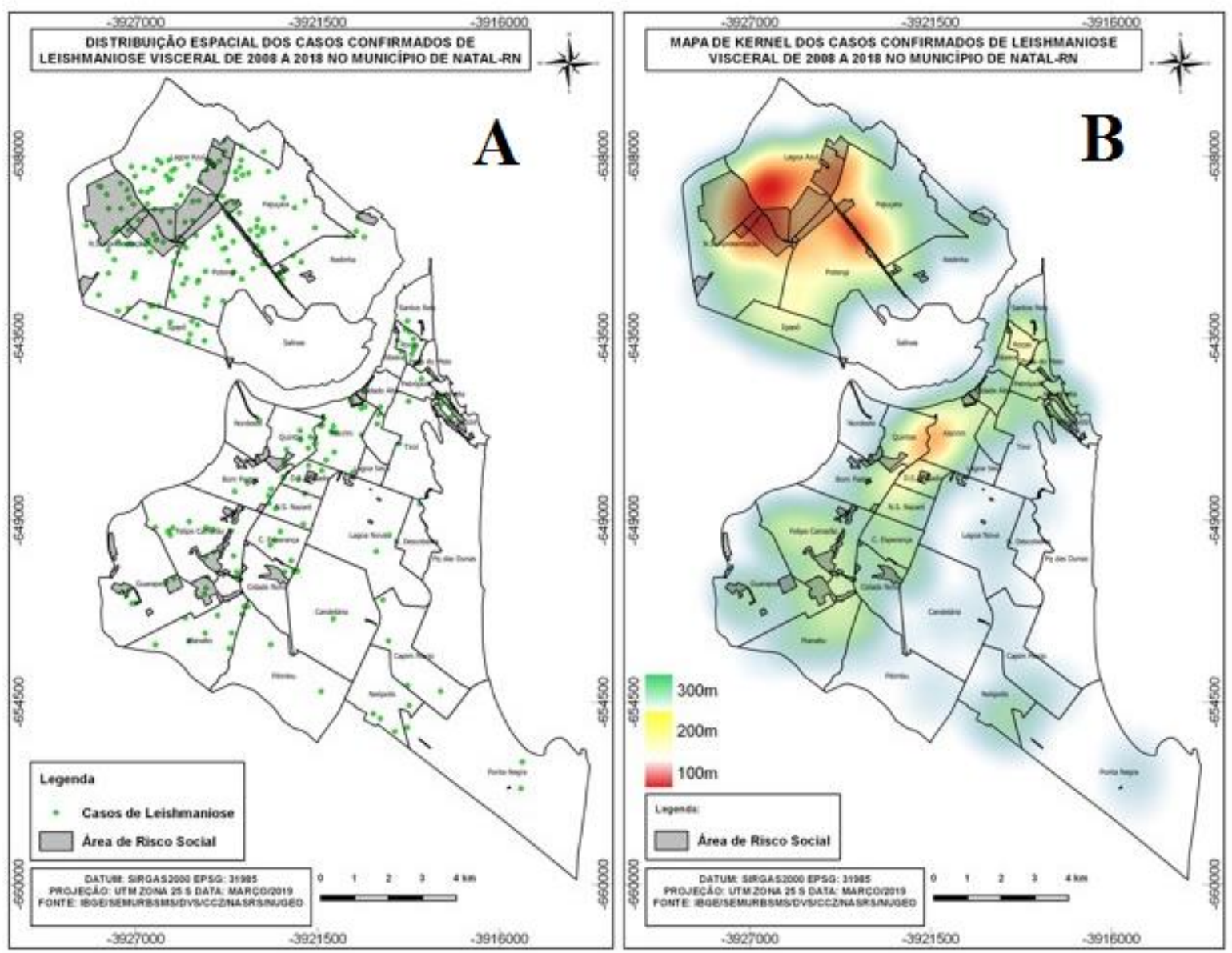

Figura 2A: Georreferenciamento dos casos de Leishmaniose visceral humana ocorridos de 2008 a 2018 no município de Natal-RN. Figura 2B: Mapa de densidade Kernel dos casos de Leishmaniose visceral humana ocorridos de 2008 a 2018, sobrepostos às áreas de Risco social no município de Natal-RN

Fonte: SINAN, 2018.

A análise da média de distância para a série histórica dos 11 anos, compreendidos entre 2008 e 2018, apresentou uma distância média de 445 metros entre o caso e a área de risco mais próxima. Observa-se que no período de 2008 e 2013 houve uma redução da distância média, e no período de 2014 a 2018, os casos se afastaram das áreas de risco. A menor distância média foi registrada no ano de 2012 (227 metros) e a maior foi em 2017 (677 metros) (Figura 5). Esse resultado demonstra que as áreas prioritárias para a realização das ações de controle da doença estão em um raio que se estendem, no máximo, a $1 \mathrm{~km}$ desde as bordas de cada área de risco. 
Figura 05: Distância média anual (DMA) entre a localização dos casos de Leishmaniose Visceral Humana e a área de Risco social mais próxima. Natal-RN, 2019

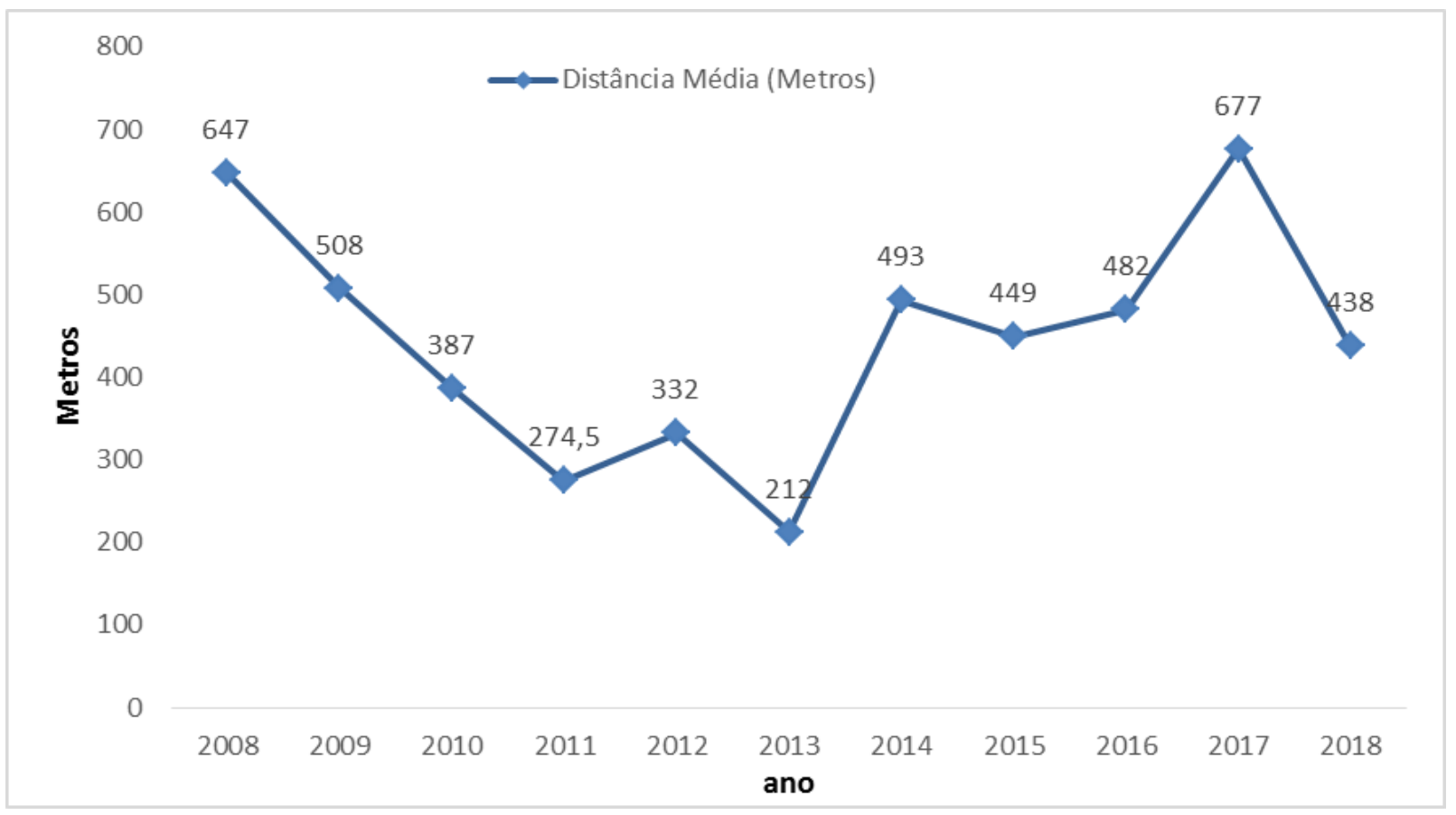

Fonte: SINAN, 2018.

\section{DISCUSSÃO}

Os resultados do presente estudo demonstram que à ocorrência de densidade dos casos de Leishmaniose visceral humana na cidade de Natal-RN possui relação de proximidade territorial com as áreas de risco social, apontando também para uma maior endemicidade nos distritos Norte e Oeste, zonas com maior número dessas áreas de risco.

Das desigualdades sociais decorrem diversos resultados, dentre os mais significativos estão às iniquidades em saúde, reflexo das desigualdades na dinâmica social e econômica, promotora da pobreza e da vulnerabilidade social. Dessa forma, os padrões sociais e a infraestrutura sanitária deficiente desempenham uma nítida interface com a situação de saúde e com as condições de vida das populações dos países em desenvolvimento, nos quais às doenças infecciosas continuam sendo uma importante causa de morbidade e mortalidade (CALIJURI et al., 2009).

O cenário epidemiológico da LVH encontrado nesse estudo está em consonância com outros trabalhos brasileiros que buscaram detectar e compreender o padrão de distribuição desse agravo, a exemplo da pesquisa realizada por Gonçalves et al. (2016) que utilizaram a análise de Kernel e geoprocessamento de dados para avaliar a distribuição da LV no município de Santarém-PA, sendo possível evidenciar que as mudanças ambientais, as migrações e a adaptação dos flebotomíneos aos centros urbanos, mudaram o padrão de adoecimento da população. O mesmo também pôde ser visto em estudo realizado por Mendes et al. (2016) em Belo Horizonte-MG.

Além da persistência das antigas áreas de ocorrência da doença, a expansão da leishmaniose visceral humana no Brasil tem sido identificado em áreas antes indenes, com o surgimento de novos focos: Rio de Janeiro (RJ), Corumbá (MS), Araçatuba (SP), Palmas (TO), Três Lagoas (MS), Campo Grande (MS), dentre outros centros urbanos (BRASIL, 2014). A proximidade entre as habitações, a alta densidade demográfica, mudanças no ambiente, como alterações climáticas e desmatamento, além de migração rural para áreas urbanas periféricas e as precárias condições de moradia e higiene contribuem para a rápida expansão da doença (LIMA et al., 2017). 
O processo de crescente urbanização e às condições de vida precárias são responsáveis por gerarem distintos padrões epidemiológicos de disseminação da LVH. Dentre esses padrões, identifica-se o padrão periurbano restrito, onde os surtos da doença são restritos as áreas de periferias urbanas, e o padrão periurbano difuso, onde os surtos da doença atingem predominantemente as periferias urbanas e, secundariamente, áreas centrais/urbanizadas (TOLEDO et al., 2017).

Os resultados do presente estudo demonstram que, na cidade de Natal, os dois padrões de disseminação da LVH citados acima foram verificados, com o maior número de casos registrados nas áreas periféricas do distrito Norte, seguida de expansão para áreas periféricas do distrito oeste e das áreas mais pobre do distrito leste, e por último, para o distrito sul da cidade.

Para compreender a expansão da LVH no município de Natal, é necessário entender o processo de crescimento urbano e a ocupação do território que se processou no município a partir da década de 1980. O processo de crescimento urbano em Natal foi provocado por um intenso movimento migratório do campo para a cidade, inicialmente porque as cidades interioranas não são capazes de reter a população local, por falta de emprego e pela falta de políticas públicas voltadas para a geração de emprego, melhoria da educação, saúde, habitação e lazer (SILVA, 2001). Esse processo se deu de forma desordenada resultando em graves problemas sociais, como: altos índices de desempregos e subempregos, o surgimento de inúmeras ocupações subnormais, caracterizadas pela precariedade das condições de vida e de saúde (GOMES; ARAÚJO, 2012).

A expansão metropolitana da cidade de Natal conformou espaços desiguais nas Regiões Administrativas da cidade, com o surgimento de uma região sul-rico e região norte-pobre que interferiram na qualidade de vida da população (SILVA; BASTOS, 2013). Essa perspectiva é corroborada pela análise realizada por Araújo e Cândido (2015) que pesquisaram a qualidade de vida nos bairros de Natal, utilizando indicadores de condições socioeconômicas e de infraestrutura urbana e ambiental, identificando nos resultas que os bairros com menor qualidade de vida são os bairros de Nossa Senhora da Apresentação, Igapó, Lagoa Azul, no distrito norte da cidade, e os bairros de Planalto, Felipe Camarão e Guarapes, na Zona Oeste da cidade. Os melhores índices foram obtidos pelos bairros do distrito sul da cidade.

A pesquisa que produziu o Índice de Vulnerabilidade Social do município de Natal revelou desigualdade significativa nas condições socioeconômicas da população de Natal, onde foram consideradas zonas de baixa vulnerabilidade os distritos das zonas sul e leste da cidade, enquanto as áreas de elevada vulnerabilidade localizam-se nas áreas periféricas das zonas oeste e norte, considerando na proposição deste índice sintético os indicadores de capital humano, capital social e de infraestrutura urbana (BARBOSA; GONÇALVES; SANTANA, 2019). Dados mais recentes destacam que os distritos Norte e Oeste da cidade de Natal são tidos como os mais populosos e que possuem rendimento nominal médio mensal, em salários, abaixo da média do município, que é de 1,78 salários mínimos (SEMURB, 2017).

Sob o prisma dessa discussão, essas evidências justificam-se os resultados do presente estudo e demonstram que as áreas de risco de elevada densidade de casos nas áreas periféricas do município e nas proximidades das áreas de risco apontam que a precariedade nas condições de infraestrutura urbana em grande parte da área analisada desempenha uma nítida interface com a situação de saúde.

Os dados expostos nesta pesquisa, bem como, estudos recentes demonstram que têm se redesenhado os limites das áreas metropolitanas e discutindo como as economias e as transformações sociais agravaram a intensidade e perversão da vulnerabilidade social e seu efeito sobre a saúde das populações. $\mathrm{O}$ alto número de famílias que vivem miseravelmente nas áreas periféricas da cidade de Natal indica que o desenvolvimento de décadas recentes não foi suficientemente inclusivo em termos de oportunidades de trabalho digno para a parte mais pobre da população (BARBOSA; GONÇALVES; SANTANA, 2019). Os resultados desse estudo confirmam que os riscos ambientais afetam diretamente aqueles que são mais vulneráveis socialmente (COSTA, 2009). 
A prevenção e o controle da leishmaniose visceral ainda é um desafio enfrentado pelos sistemas de saúde, principalmente dos países pobres e em desenvolvimento. O Ministério da Saúde define no Programa de Vigilância e Controle da Leishmaniose Visceral que como ferramentas de controle a LVH três medidas principais: destruição do inseto vetor, tratamento dos casos humanos e eliminação dos reservatórios (cães sintomáticos e soropositivos) (BRASIL, 2006). Contudo, observa-se pouco investimento na realização de campanhas sistemáticas de educação em saúde e esclarecimento à população sobre as formas de prevenção e controle. Os programas de controle da leishmaniose visceral ainda estão centrados na eutanásia de cães soropositivos, e sempre alegam questões financeiras para o investimento necessário no controle dessa endemia (CASTRO et al., 2016).

É importante destacar que as ações de educação em saúde e de mobilização social desempenham um papel essencial no âmbito da Vigilância e Controle da LVH, na medida em que se constitui de potencial transformador da construção de novos saberes e fazeres da sociedade. Esses dois componentes devem ser reconhecidos e incorporados em cada serviço local, na perspectiva de ações e práticas de saúde participativas, educativas, comprometidas com a transformação de indivíduos em sujeitos, de grupos de indivíduos em sujeitos coletivos, colaborando para aumentar a consciência e compreensão sobre as condições de vida e as relações existentes com a saúde (RANGEL et al., 2013). Embora seja atribuído um papel de grande relevância às atividades de educação em saúde, elas ainda são pouco valorizadas dentro do contexto dos serviços de vigilância em saúde (ZUBEN; DONALíSIO, 2016).

Os resultados da presente pesquisa permitiram identificar as áreas geográficas de maior densidade, se sobrepõem ao índice de risco para ocorrência da LVH no município de Natal e destacaram a importância das áreas de risco social para a dinâmica desta endemia. A identificação dessas áreas possibilita a escolha de estratégias e intervenções específicas bem como a priorização de recursos para os grupos mais vulneráveis, contribuindo assim para diminuir as iniquidades em saúde.

Os estudos realizados a partir dos Sistemas de Informações Geográficas (SIG), ferramentas computacionais do campo do geoprocessamento, são recursos que, quando aplicados às questões de saúde coletiva, representam uma ferramenta que permite para o mapeamento de doenças, a avaliação de riscos, o planejamento de ações e análise de ações, baseado na distribuição espacial e temporal das ocorrências da doença (BARCELLOS; BASTOS, 1996). Assim, estudos de distribuição e análise espacial têm sido ferramentas úteis que podem contribuindo substancialmente com as politicas públicas e mais diretamente à vigilância em saúde (LUCENA et al., 2012).

Por tratar-se de uma abordagem ecológica, o estudo apresenta limitações inerentes à sua metodologia, com relação à utilização das informações obtidas em bancos de dados secundários, estando sujeito ao viés da subnotificação. Apesar dessas limitações, esse estudo foi útil no levantamento de possíveis fatores ambientais elencados no estudo e associados à difusão da LVH no espaço urbano, assim como na indicação de áreas prioritárias para o desenvolvimento de ações de controle da doença.

Esse estudo reforça que o cenário epidemiológico do município de Natal-RN indica a necessidade de realização de ações de saúde para o controle da LVH no entorno das áreas de risco social, bem como a implementação de políticas de assistência social, habitação e infraestrutura urbana, com investimento na melhoria das condições de vida das populações negligenciadas, focando principalmente na redução do número de indivíduos que habitam em condições precárias e que ocupam estas áreas de risco.

\section{CONCLUSÕES}

A distribuição da LVH em Natal-RN apresentou a ocorrência em padrões de densidade nas áreas periféricas, ocupadas de forma desordenada, com infraestrutura pública deficiente e condições de vida desfavoráveis. A partir desses achados, faz-se necessário ponderar os aspectos políticos, éticos, econômicos, sociais, ecológicos, culturais e outros para que se obtenha uma visão global do problema e de suas alternativas e soluções. As medidas de controle da LVH no município precisam ser readequadas, sendo necessário investimento em medidas de proteção eficaz para o controle do vetor e melhorias na organização dos espaços urbanos priorizando o crescimento planejado e boas DOI: http://dx.doi.org/10.14393/Hygeia153249966 Hygeia 15 (31): 121 - 133, Junho/2019 página 130 
Padrões espaciais da ocorrência de leishmaniose visceral humana na cidade de Natal-RN: a influência das áreas de risco social
Ana Mayara Gomes de Souza

Talita Araujo de Souza

Isabelle Ribeiro Barbosa

condições de infraestrutura. Além disso, as ações também devem ter como foco a educação em saúde.

Assim, entende-se que ainda há muito que se conhecer sobre o perfil epidemiológico da LVH e o seu processo de urbanização na cidade de Natal, mas o referido estudo alcançou o objetivo proposto permitindo conhecer a dinâmica de ocorrência e expansão da leishmaniose visceral nos distritos do município, podendo servir de base para futuras ações de controle endêmico, assim como despertar o desenvolvimento de novos estudos nesta temática a fim de identificar as áreas de maior vulnerabilidade para disseminação e manutenção da LV.

\section{REFERÊNCIAS}

ARAÚJO, M.C.C.; CÂNDIDO, G.A. Índices de qualidade de vida urbana de Natal-RN. Geoconexões, v. 1, n. 1, p. 51-66, 2015. Disponível em: < http://www2.ifrn.edu.br/ojs/index.php/geoconexoes/article/view/2836>. Acesso em: 11 jul. 2019. https://doi.org/10.15628/geoconexoes.2015.2836

BARBOSA, I.R. Epidemiologia da Leishmaniose Visceral no estado do Rio Grande do Norte, Brasil. Revista de Epidemiologia e Controle de Infecção, v. 3, n. 1, p. 17-21, 2013. Disponível em: $<$ https://online.unisc.br/seer/index.php/epidemiologia/article/view/3148>. Acesso em: 07 ago. 2019. https://doi.org/10.17058/reci.v3i1.3148

BARBOSA, I.R. Leishmaniose visceral humana no município de Natal-RN: análise clínicoepidemiológica e espacial. Revista Ciência Plural, v. 2, n. 1, p. 89-101, 2016. Disponível em: $<$ https://periodicos.ufrn.br/rcp/article/view/8559>. Acesso em: 15 jul. 2019.

BARBOSA, I.R.; GONÇALVES, R.C.B.; SANTANA, R.L. Mapa de vulnerabilidade social para o Município de Natal (Nordeste do Brasil) em nível de setor censitário. J Hum Crescimento Dev. v.29, n.1, p. 48-56, 2019. Disponível em: <https://doi.org/10.7322/jhgd.157749>. Acesso em: 11 jul. 2019.

BARCELLOS, C; BASTOS, F.I. Geoprocessamento, ambiente e saúde: uma união possível?. Cadernos de Saúde Pública, v. 12, p. 389-397, 1996. Disponível em: $<$ https://www.scielosp.org/scielo.php?pid=S0102-311X1996000300012\&script=sci abstract $>$. Acesso em: 07 ago. 2019. https://doi.org/10.1590/S0102-311X1996000300012

Brasil. Ministério da Saúde. Secretaria de Vigilância em Saúde. Departamento de Vigilância Epidemiológica. Manual de vigilância e controle da leishmaniose visceral / Ministério da Saúde, Secretaria de Vigilância em Saúde, Departamento de Vigilância Epidemiológica. - Brasília: Editora do Ministério da Saúde, 2006. 120 p. Disponível em: <

https://www.paho.org/bra/index.php?option=com docman\&view=download\&alias=1462-manualvigilancia-e-controle-da-leishmaniose-visceral-2\&category slug=leishmanioses-978\&ltemid=965> Acesso em: 06 setembro 2019.

BRASIL. Ministério do Desenvolvimento Agrário; Secretaria de Desenvolvimento Territorial. Plano territorial de desenvolvimento rural sustentável do Baixo Tocantins. Distrito Federal, número 1, p. 83. 2015.

BRASIL. Ministério da Saúde. Secretaria de Vigilância em Saúde. Departamento de Vigilância Epidemiológica. Leishmaniose visceral: recomendações clínicas para redução da letalidade. Brasília: Ministério da Saúde, 2011.

CALIJURI, M.L et al. Estudo de indicadores de saúde ambiental e de saneamento em cidade do Norte do Brasil. Eng Sanit Ambient, v. 14, n. 1, p. 19-28, 2009. Disponível em: < http://www.scielo.br/pdf/esa/v14n1/v14n1a03>. Acesso em: 07 ago. 2019. https://doi.org/10.1590/S1413-41522009000100003

CARDIM, M.F.M et al. Introdução e expansão da Leishmaniose visceral americana em humanos no estado de São Paulo, 1999-2011. Revista de Saúde Pública, v. 47, p. 691-700, 2013. Disponível em: $<$ https://www.scielosp.org/scielo.php?pid=S0034-89102013000600691\&script=sci arttext\&tlng=en>. Acesso em: 15 jul. 2019. https://doi.org/10.1590/S0034-8910.2013047004454 
CARDIM, M.F.M et al. Leishmaniose visceral no estado de São Paulo, Brasil: análise espacial e espaço-temporal. Revista de Saúde Pública, v. 50, p. 48-48, 2016. Disponível em: $<$ https://www.revistas.usp.br/rsp/article/view/126538>. Acesso em: 10 jun. 2019.

CASTRO, J.M et al. Conhecimento, percepções de indivíduos em relação à leishmaniose visceral humana como novas ferramentas de controle. Ensaios e Ciência: C. Biológicas, Agrárias e da Saúde, v. 20, n. 2, p. 93-103, 2016. Disponível em:

$<$ http://revista.pgsskroton.com.br/index.php/ensaioeciencia/article/view/3540>. Acesso em: 05 ago. 2019. https://doi.org/10.17921/1415-6938.2016v20n2p93-103

CAVALCANTE, Í.J.M.; VALE, .R. Epidemiological aspects of visceral leishmaniasis (kala-azar) in Ceará in the period 2007 to 2011. Revista Brasileira de Epidemiologia, v. 17, p. 911-924, 2014. Disponível em: < https://www.scielosp.org/article/rbepid/2014.v17n4/911-924/pt/>. Acesso em: 10 jun. 2019. https://doi.org/10.1590/1809-4503201400040010

CHIARAVALLOTI-NETO, F. O geoprocessamento e saúde pública. Arquivos de Ciências da Saúde, v. 23, n. 4, p. 01-02, 2017. Disponível em: <

http://www.cienciasdasaude.famerp.br/index.php/racs/article/view/661>. Acesso em: 19 jul. 2019.

COSTA, M.C.L.; DANTAS, E.W.C. Vulnerabilidade socioambiental na região metropolitana de

Fortaleza. Eustogio Wanderely Correia, 2009. https://doi.org/10.17696/2318-3691.23.4.2016.661

D'ANDREA, L.A.Z; GUIMARÃES, R.B. A importância da análise de distribuição espacial da leishmaniose visceral humana e canina para as ações de vigilância em saúde. Hygeia, v. 14, n. 28, p. 121-138, 2018. Disponível em: <http://www.seer.ufu.br/index.php/hygeia/article/view/41515>. Acesso em: 15 jul. 2019.

GOMES, R.C.C.; ARAÚJO, J.C. O Estado e sua intervenção no urbano no Rio Grande do Norte. Sociedade e Território, p. 59-76, 2012. Disponível em: < https://periodicos.ufrn.br/sociedadeeterritorio/article/view/3464>. Acesso em: 11 jul. 2019.

GONÇALVES, R et al. Diversity and ecology of sand flies (Psychodidae: Phlebotominae): foci of cutaneous leishmaniasis in Amazon Region, Brazil. Revista Pan-Amazônica de Saúde, v. 7, n. esp, p. 133-142, 2016. Disponível em: <http://scielo.iec.gov.br/scielo.php?pid=S217662232016000500133\&script=sci abstract>. Acesso em: 07 ago. 2019. https://doi.org/10.5123/S2176$\underline{62232016000500015}$

LIMA, A. L. M. Fatores associados a infecção humana por Leishmania infantum e seu padrão espacial em uma área endêmica do Nordeste do Brasil. 2017. 65f. Tese (Doutorado em Ciências da Saúde) Centro de Ciências da Saúde, Universidade Federal do Rio Grande do Norte, Natal, 2017.

LUCENA, K.D.T et al. Análise espacial da violência doméstica contra a mulher entre os anos de 2002 e 2005 em João Pessoa, Paraíba, Brasil. Cadernos de Saúde Pública, v. 28, p. 1111-1121, 2012. Disponível em: <https://www.scielosp.org/article/csp/2012.v28n6/1111-1121/pt/>. Acesso em: 07 ago. 2019. https://doi.org/10.1590/S0102-311X2012000600010

MENDES, C.S et al. Impacto das mudanças climáticas sobre a leishmaniose no Brasil. Ciência \& Saúde Coletiva, v. 21, p. 263-272, 2016. Disponível em: < https://www.scielosp.org/article/csc/2016.v21n1/263-272/pt/>. Acesso em: 07 ago. 2019. https://doi.org/10.1590/1413-81232015211.03992015

RANGEL, O et al. Classificação epidemiológica dos municípios segundo o Programa de Vigilância e Controle da Leishmaniose Visceral Americana no estado de São Paulo, para 2013. BEPA. Boletim Epidemiológico Paulista (Online), v. 10, n. 111, p. 3-14, 2013. Disponível em: < http://periodicos.ses.sp.bvs.br/scielo.php?script=sci arttext\&pid=S180642722013000300002\&lng=pt\&nrm=isso $>$. Acesso em: 05 ago. 2019.

SEMURB. Prefeitura Municipal de Natal. Secretaria Municipal de Meio Ambiente e Urbanismo SEMURB. Plano Diretório de Natal/Conheça Melhor seu Bairro. Versão 1. 2017.

SILVA, A. F. C.; BASTOS, N.S.M. Novas (e velhas) fronteiras: os espaços de pobreza e a expansão da cidade de Natal-RN. Anais: Encontros Nacionais da ANPUR, v. 10, 2013. 
SILVA, A.F. Migração e crescimento urbano: uma reflexão sobre a cidade de Natal, Brasil. Scripta Nova: revista electrónica de geografía y ciencias sociales, v. 5, 2001. Disponível em: < https://www.raco.cat/index.php/ScriptaNova/article/view/58998>. Acesso em: 11 jul. 2019.

SILVA, K.B.M et al. ANÁLISE ESPACIAL DA LEISHMANIOSE VISCERAL NO MUNICÍPIO DE PALMAS, TOCANTINS, BRASIL. Hygeia: Revista Brasileira de Geografia Médica e da Saúde, v. 13, n. 25, p. 18, 2017. Disponível em: < https://patua.iec.gov.br/handle/iec/3681 >. Acesso em: 15 jul. 2019. https://doi.org/10.14393/Hygeia132502

TOLEDO, C.R.S et al. Vulnerabilidade à transmissão da leishmaniose visceral humana em área urbana brasileira. Revista de Saúde Pública, v. 51, p. 1-11, 2017. Disponível em: < https://www.redalyc.org/pdf/672/67249591047.pdf>. Acesso em: 10 jun. 2019.

ZUBEN, A.P.B.V.; DONALÍSIO, M.R. Dificuldades na execução das diretrizes do Programa de Vigilância e Controle da Leishmaniose Visceral em grandes municípios brasileiros. Cadernos de Saúde Pública, v. 32, p. e00087415, 2016. Disponível em:

$<$ https://www.scielosp.org/scielo.php?pid=S0102-311X2016000600401\&script=sci arttext\&tlng=pt $>$. Acesso em: 19 jul. 2019. https://doi.org/10.1590/0102-311X00087415

DNDi. Drugs for Neglected Diseases initiative. Visceral Leishmaniasis Disease Background. Disponível em: < https://www.dndi.org/diseases-projects/leishmaniasis/disease-background-vl/>. Acessado em: 06 de set.2019. 